\title{
Polynomial Expansions of Bessel Functions and Some Associated Functions
}

\author{
By Jet Wimp
}

1. Introduction. In this paper we first determine representations for the AngerWeber functions $\mathrm{J}_{v}(a x)$ and $\mathrm{E}_{v}(a x)$ in series of symmetric Jacobi polynomials. (These include Legendre and Chebyshev polynomials as special cases.) If $v$ is an integer, these become expansions for the Bessel function of the first kind, since $\mathrm{J}_{k}(a x)=J_{k}(a x)$. In Section 3, corresponding representations are found for $(a x)^{-v} J_{v}(a x)$. Convenient error bounds are obtained for the above expansions. In the fourth section we determine the similar type expansions for the Bessel functions $Y_{k}(a x)$ and $K_{k}(a x)$. In Section 5, the coefficients of some of our expansions are tabulated for particularly important values of the various parameters.

2. Symmetric Jacobi Expansions of Anger-Weber Functions. A function $f(x)$ satisfying certain conditions (for these consult [1]) may be expanded in the series

$$
f(x)=\sum_{n=0}^{\infty} C_{n} P_{n}^{(\alpha, \alpha)}(x), \quad-1 \leqq x \leqq 1, \quad \alpha>-1,
$$

where $P_{n}^{(\alpha, \alpha)}(x)$ is called the symmetric Jacobi polynomial of degree $n$. For our present purposes we shall use a definition given in [2]:

$$
2^{n} n ! P_{n}^{(\alpha, \alpha)}(x)=(-)^{n}\left(1-x^{2}\right)^{-\alpha} D^{n}\left[\left(1-x^{2}\right)^{\alpha+n}\right] .
$$

Also

$$
\begin{gathered}
C_{n}=h_{n}^{-1} \int_{-1}^{1} f(x)\left(1-x^{2}\right)^{\alpha} P_{n}^{(\alpha, \alpha)}(x) d x \\
h_{n}=\frac{2^{2 \alpha}(n+1)_{\alpha}}{\left(n+\alpha+\frac{1}{2}\right)(n+\alpha+1)_{a}} ; \quad(v)_{\mu}=\frac{\Gamma(v+\mu)}{\Gamma(v)}, \quad(v)_{0}=1 .
\end{gathered}
$$

Using the representation (2.2) in (2.3) and noticing that all derivatives of $\left(1-x^{2}\right)^{\alpha+n}$ up to and including the $(n-1)$ st vanish at $x= \pm 1$, we integrate (2.3) $n$ times by parts to get:

$$
C_{n}=\left(2^{n} n ! h_{n}\right)^{-1} \int_{-1}^{1} f^{(n)}(x)\left(1-x^{2}\right)^{\alpha+n} d x
$$

Consider the integral definition of the Anger-Weber functions [2, v. 2, p. 35]

$$
\mathrm{J}_{v}(a x)+i \mathrm{E}_{v}(a x)=\pi^{-1} \int_{0}^{\pi} e^{i[v \phi-a x \sin \phi]} d \phi=f(x) .
$$

When $v$ is an integer, $\mathrm{J}_{v}(a x)$ coincides with the Bessel function of the firsu kind $J_{v}(a x)[2$, v. 2, p. 4].

Now differentiate (2.6) $n$ times under the integral sign, substitute the result in

Received February 27, 1962. 
(2.5) and interchange the order of integration (which is, of course, permissible). The inner integral is known [3] and after evaluating it we have

$$
C_{n}=(-i)^{n}(n+1)_{\alpha}\left(h_{n} \pi^{1 / 2}\right)^{-1}
$$

$$
\cdot \int_{0}^{\pi} e^{i v \phi}\left\{\frac{a \sin \phi}{2}\right\}^{-[\alpha+(1 / 2)]} J_{[n+\alpha+(1 / 2)]}(a \sin \phi) d \phi .
$$

Use the power series expansion for the Bessel function in (2.7) and integrate termby-term to get

$$
C_{n}=(-i)^{n}\left[\cos \frac{v \pi}{2}+i \sin \frac{v \pi}{2}\right] \Lambda_{n} R_{n}(v, \alpha, a),
$$

where

$$
\Lambda_{n}=\frac{a^{n} n !}{\Gamma\left(\frac{n}{2}+\frac{v}{2}+1\right) \Gamma\left(\frac{n}{2}-\frac{v}{2}+1\right)(n+2 \alpha+1)_{n}}
$$

and $R_{n}$ is conveniently described in hypergeometric notation [2, v. 1, p. 182] as

$$
\begin{aligned}
R_{n}(v, \alpha, a)={ }_{2} F_{3}\left[\frac{n}{2}+\frac{1}{2},\right. & \frac{n}{2}+1 \\
& \left.\alpha+n+\frac{3}{2}, \frac{n}{2}+\frac{v}{2}+1, \frac{n}{2}-\frac{v}{2}+1 ;-\frac{a^{2}}{4}\right]
\end{aligned}
$$

Equating real and imaginary parts of (2.6) and (2.1), we get

$$
\begin{aligned}
& \mathrm{J}_{v}(a x)=\sum_{n=0}^{\infty} A_{n} P_{n}^{(\alpha, \alpha)}(x),-1 \leqq x \leqq 1, \\
& \mathbf{E}_{v}(a x)=\sum_{n=0}^{\infty} B_{n} P_{n}^{(\alpha, \alpha)}(x), \quad-1 \leqq x \leqq 1,
\end{aligned}
$$

where

$$
\begin{aligned}
& A_{n}=\Lambda_{n} R_{n}(v, \alpha, a) \phi_{n}(v), \\
& B_{n}=\Lambda_{n} R_{n}(v, \alpha, a) \psi_{n}(v),
\end{aligned}
$$

and

$$
\begin{aligned}
& \phi_{n}(v)=\left\{\begin{array}{lll}
(-)^{n / 2} \cos \frac{v \pi}{2}, & n & \text { even }, \\
(-)^{(n-1) / 2} \sin \frac{v \pi}{2}, & n & \text { odd }
\end{array}\right. \\
& \psi_{n}(v)=\left\{\begin{array}{lll}
(-)^{n / 2} \sin \frac{v \pi}{2}, & n & \text { even }, \\
(-)^{(n+1) / 2} \cos \frac{v \pi}{2}, & n & \text { odd }
\end{array}\right.
\end{aligned}
$$

Equations (2.11) and (2.12) and the expansions in Section 3 may also be de- 
rived from results in [4]. The present derivation is more satisfactory because it establishes a foundation for the work in Section 4.

When $\alpha=-\frac{1}{2}$,

$$
P_{n}^{[-(1 / 2),-(1 / 2)]}(x)=\left(\frac{1}{2}\right)_{n}(n !)^{-1} T_{n}(x), \quad n=1,2 \cdots,
$$

where $T_{n}(x)$ is the Chebyshev polynomial of the first kind of degree $n$. Also for this value of $\alpha, R_{n}$ simplifies to the product of two Bessel functions [2, v. 2, p. 11]. With $\alpha=-\frac{1}{2}$, then (2.11) $-(2.14)$ become

$$
\begin{aligned}
& \mathrm{J}_{v}(a x)=\sum_{n=0}^{\infty} C_{n} T_{n}(x), \quad-1 \leqq x \leqq 1, \\
& \mathrm{E}_{v}(a x)=\sum_{n=0}^{\infty} D_{n} T_{n}(x), \quad-1 \leqq x \leqq 1,
\end{aligned}
$$

where

$$
\begin{aligned}
& C_{n}=\epsilon_{n} J_{(n+v) / 2}\left(\frac{a}{2}\right) J_{(n-v) / 2}\left(\frac{a}{2}\right) \phi_{n}(v), \\
& D_{n}=\epsilon_{n} J_{(n+v) / 2}\left(\frac{a}{2}\right) J_{(n-v) / 2}\left(\frac{a}{2}\right) \psi_{n}(v),
\end{aligned}
$$

and $\epsilon_{n}=\left\{\begin{array}{l}1, n=0 \\ 2, n>0\end{array}\right.$

For integral $v$ we have the expansions

$$
\begin{gathered}
J_{2 k}(a x)=\sum_{n=0}^{\infty} \epsilon_{n} J_{k+n}\left(\frac{a}{2}\right) J_{k-n}\left(\frac{a}{2}\right) T_{2 n}(x),-1 \leqq x \leqq 1, \\
J_{2 k+1}(a x)=2 \sum_{n=0}^{\infty} J_{k+n+1}\left(\frac{a}{2}\right) J_{k-n}\left(\frac{a}{2}\right) T_{2 n+1}(x),-1 \leqq x \leqq 1,
\end{gathered}
$$

and $k=0,1,2 \cdots$. Equation (2.22) is known [2, v. 2, p. 100].

Since

$$
J_{v}(i z)=e^{(v \pi i) / 2} I_{v}(z),
$$

where $I_{v}(z)$ is the modified Bessel function of the first kind [2, v. 2, p. 5], we may replace $a$ by $i a$ in (2.22) and (2.23) to get expansions for $I_{2 k}(a x)$ and $I_{2 k+1}(a x)$.

It is important to note that, although the above expansions are valid only for $x$ real and $|x| \leqq 1,(2.6)$ is entire in $a$ and $v$, and hence $a$ may be chosen arbitrarily to yield expansions valid anywhere in the finite complex plane.

The expansions (2.11), (2.12), (2.18), (2.19), (2.22), and (2.23) are quite rapidly convergent, particularly in the Chebyshev cases [5]; consequently the last four expansions are eminenily suitable for use on digital computers. ${ }^{*}$ Such series

* The Bessel functions required to compute the coefficients in our expansions can be systematically generated on electronic computers with the aid of techniques discussed in $[6,7,8]$. There are numerous tables available for hand calculations. The words "accuracy," "error," and "convergence" in this paper always refer to the properties of the expansion when truncated after a finite number of terms. 
may be truncated and rearranged in powers of $x$. Clenshaw [9], though, by using the recursion formulas satisfied by the Chebyshev polynomials, has formulated a convenient nesting procedure which allows one to utilize such expansions directly. The scheme is as follows. Consider

$$
\begin{aligned}
f^{(1)}(x) & =\sum_{n=0}^{N} A_{n}{ }^{(1)} T_{n}{ }^{*}\left(\frac{x}{a}\right), \quad 0 \leqq x \leqq a, \\
f^{(2)}(x) & =\sum_{n=0}^{N} A_{n}{ }^{(2)} T_{2 n}\left(\frac{x}{a}\right), \quad-a \leqq x \leqq a, \\
f^{(3)}(x) & =\sum_{n=0}^{N} A_{n}{ }^{(3)} T_{2 n+1}\left(\frac{x}{a}\right), \quad-a \leqq x \leqq a .
\end{aligned}
$$

To evaluate the series (2.25), (2.26), or (2.27), respectively, we construct the following sequences:

$$
\begin{aligned}
& {b_{n}}^{(1)}=\left[4\left(\frac{x}{a}\right)-2\right] b_{n+1}^{(1)}-b_{n+2}^{(1)}+A_{n}{ }^{(1)}, \\
& b_{n}{ }^{(2)}=\left[4\left(\frac{x}{a}\right)^{2}-2\right] b_{n+1}^{(2)}-b_{n+2}^{(2)}+A_{n}{ }^{(2)}, \\
& b_{n}{ }^{(3)}=\left[4\left(\frac{x}{a}\right)^{2}-2\right] b_{n+1}^{(3)}-b_{n+2}^{(3)}+A_{n}{ }^{(3)},
\end{aligned}
$$

for $n=N, N-1, N-2, \cdots, 3,2,1,0$ with the initial values

$$
b_{N+1}^{(1)}=b_{N+2}^{(1)}=b_{N+1}^{(2)}=b_{N+2}^{(2)}=b_{N+1}^{(3)}=b_{N+2}^{(3)}=0 \text {. }
$$

$f^{(1)}(x), f^{(2)}(x)$, and $f^{(3)}(x)$ are then given by

$$
\begin{aligned}
& f^{(1)}(x)=b_{0}{ }^{(1)}+b_{1}{ }^{(1)}\left[1-2\left(\frac{x}{a}\right)\right] \\
& f^{(2)}(x)=b_{0}{ }^{(2)}+b_{1}{ }^{(2)}\left[1-2\left(\frac{x}{a}\right)^{2}\right], \\
& f^{(3)}(x)=\left[b_{0}{ }^{(3)}-b_{1}{ }^{(3)}\right]\left(\frac{x}{a}\right) .
\end{aligned}
$$

The method is as direct as the ordinary nesting process used to evaluate polynomials.

We now derive error estimates for the expansions (2.11) and (2.12) for $-1 \leqq x \leqq 1$. Notice that

$$
R_{n}(v, \alpha, a)=1+0\left(\frac{1}{n}\right)
$$

provided all other parameters are fixed, and consequently

$$
\left|A_{n}\right| \leqq \frac{|a|^{n} n !}{\Gamma\left(\frac{n+v}{2}+1\right) \Gamma\left(\frac{n-v}{2}+1\right)(n+2 \alpha+1)_{n}}\left|1+0\left(\frac{1}{n}\right)\right|
$$


and likewise for $B_{n}$. Also [:, v. 2, p. 206]

$$
\max _{-1 \leqq x \leqq 1}\left|P_{n}^{(\alpha, \alpha)}(x)\right|=\left(\begin{array}{c}
n+\alpha \\
n
\end{array}\right), \quad \alpha \geqq-\frac{1}{2} .
$$

Let $\epsilon_{N}$ denote the error incurred by taking just $N$ terms of (2.11) or (2.12). Because of the rapidity of convergence of the expansions, as shown by $(2.35)$, the $(N+1)$ th term furnishes us with a convenient error estimate

$$
\left|\epsilon_{N}\right|=\frac{|a|^{N} N^{\alpha+(1 / 2)} \pi^{1 / 2}}{2^{2 N+2 \alpha} \Gamma\left(\frac{N+v}{2}+1\right) \Gamma\left(\frac{N-v}{2}+1\right) \Gamma(\alpha+1)}\left|1+0\left(\frac{1}{N}\right)\right|,
$$

where $\alpha \geqq-\frac{1}{2}, \quad N>v,-1 \leqq x \leqq 1$.

Among the values of $\alpha$ considered, it follows from (2.37) that the choice $\alpha=-\frac{1}{2}$, i.e., the Chebyshev case, yields the smallest error term for large $N$.

3. Expansions of Bessel Functions of the First Kind of Nonintegral Order. Results in the previous section gave symmetric Jacobi polynomial expansions for $J_{v}(a x)$ and $I_{v}(a x)$ for integral $v$. When $v$ is nonintegral, these functions are no longer entire functions of $x$, and it is convenient to derive an expansion for the entire function

$$
\Gamma(v+1)(a x / 2)^{-v} J_{v}(a x)={ }_{0} F_{1}\left(v+1 ;-\frac{a^{2} x^{2}}{4}\right) .
$$

Corresponding expansions for $\Gamma(v+1)(a x / 2)^{-v} I_{v}(a x)$ then follow, as before, from (2.24).

Let $f(x)$ in (2.5) be the right-hand side of (3.1). Then we have

$$
J_{v}(a x)=(a x)^{\nu} \sum_{n=0}^{\infty} A_{n} P_{2 n}^{(\alpha, \alpha)}(x), \quad-1 \leqq x \leqq 1
$$

where

$$
\begin{aligned}
A_{n}=\frac{(-)^{n}(2 a)^{2 n}}{2^{v} \pi^{1 / 2}(2 n+2 \alpha+1)_{2 n}\left(n+\frac{1}{2}\right)_{v+(1 / 2)}} & \\
& \cdot{ }_{1} F_{2}\left(n+\frac{1}{2} ; \quad v+n+1, \quad 2 n+\alpha+\frac{3}{2} ;-\frac{a^{2}}{4}\right) .
\end{aligned}
$$

These equations also follow from a result in [4]. Indeed, using a general expansion given there, an alternative formula for (3.3) can be stated. We have

$$
\begin{gathered}
{ }_{1} F_{2}\left[\rho ; \sigma, \tau ;-\frac{z^{2}}{4}\right]=\Gamma(\sigma)(z / 2)^{1-\sigma} \sum_{k=0}^{\infty} \frac{(z / 2)^{k}(\tau-\rho)_{k}}{k !(\tau)_{k}} J_{k+\sigma-1}(z), \\
A_{n}=\frac{(-)^{n} 2^{(1 / 2)-\alpha-v} \Gamma\left(n+\frac{1}{2}\right)\left(2 n+\alpha+\frac{1}{2}\right)(2 n+\alpha+1)_{\alpha}}{a^{(1 / 2)+\alpha}} \\
\cdot \sum_{k=0}^{\infty} \frac{(a / 2)^{k}\left(v+\frac{1}{2}\right)_{k}}{k ! \Gamma(v+n+k+1)} J_{2 n+k+\alpha+(1 / 2)}(a) .
\end{gathered}
$$


For the Chebyshev case of (3.2) $\alpha=-\frac{1}{2}$ and

$$
J_{v}(a x)=(a x)^{\nu} \sum_{n=0}^{\infty} C_{n} T_{2 n}(x), \quad-1 \leqq x \leqq 1,
$$

where

$$
C_{n}=\frac{\epsilon_{n}(-)^{n}(a / 4)^{2 n}}{2^{v} n ! \Gamma(v+n+1)}{ }_{1} F_{2}\left[n+\frac{1}{2} ; \quad v+n+1,2 n+1 ;-\frac{a^{2}}{4}\right] .
$$

Notice that when $v=-\frac{1}{2}$, (3.3) simplifies. Also, since

$$
J_{-(1 / 2)}(a x)=\left(\frac{\pi a x}{2}\right)^{-(1 / 2)} \cos (a x)
$$

we infer the expansion

$$
\cos (a x)=\sum_{n=0}^{\infty} C_{n} P_{2 n}^{(\alpha, \alpha)}(x), \quad-1 \leqq x \leqq 1,
$$

where

$$
C_{n}=\frac{(-)^{n} \pi^{1 / 2} 2^{-\alpha+(1 / 2)}\left(2 n+\alpha+\frac{1}{2}\right)(2 n+\alpha+1)_{\alpha}}{a^{\alpha+(1 / 2)}} J_{2 n+\alpha+(1 / 2)}(a),
$$

a formula which can be derived in a number of different ways.

Using an analysis similar to that of Section 2, we may derive the estimate for the error incurred when just $N$ terms of (3.2) are used.

$$
\begin{aligned}
&\left|\epsilon_{N}\right|=\frac{|a|^{v+2 N}|x|^{v} \pi^{1 / 2} N^{\alpha+(1 / 2)}}{2^{4 N+\alpha+v-(1 / 2)} N ! \Gamma(N+v+1) \Gamma(\alpha+1)} \mid 1+0\left(\frac{1}{N}\right) \mid, \\
&-1 \leqq x \leqq 1, \quad \alpha \geqq-\frac{1}{2}, \quad N>v .
\end{aligned}
$$

Concerning the optimum choice of $\alpha$ in (3.2), see the discussion surrounding (2.37).

4. Expansions of Bessel Functions of the Second Kind. The Bessel function and modified Bessel function of the second kind are denoted by $Y_{v}(z)$ and $K_{v}(z)$, respectively, and a treatment of them can be found in [2, v. 2, Ch. VII]. If $v$ is nonintegral, then

$$
Y_{v}(z)=[\sin (v \pi)]^{-1}\left\{J_{v}(z) \cos (v \pi)-J_{-v}(z)\right\}
$$

and

$$
K_{v}(z)=\frac{\pi}{2}[\sin (v \pi)]^{-1}\left\{I_{-v}(z)-I_{v}(z)\right\}
$$

so for such values of $v$ expansions for the functions follow directly from the results of Section 3.

If $v$ is an integer, it can be shown that

$$
Y_{k}(a x)=\frac{2}{\pi}\left[\gamma+\ln \left(\frac{a x}{2}\right)\right] J_{k}(a x)+N_{k-1}(a x)-\frac{1}{\pi} W_{k}(a x),
$$


and

$$
K_{k}(a x)=(-)^{k+1}\left[\gamma+\ln \left(\frac{a x}{2}\right)\right] I_{k}(a x)-\frac{\pi}{2} i^{k} N_{k-1}(i a x)+\frac{i^{k}}{2} W_{k}(i a x),
$$

where

$$
N_{k-1}(a x)= \begin{cases}-\frac{1}{\pi} \sum_{m=0}^{k-1}\left(\frac{a x}{2}\right)^{2 m-k} \frac{(k-m-1) !}{m !}, & k>0 \\ 0, & k=0\end{cases}
$$

and

$$
W_{k}(a x)=\sum_{m=0}^{\infty}(-)^{m}\left(\frac{a x}{2}\right)^{k+2 m} \frac{\left[h_{m+k}+h_{m}\right]}{m !(k+m) !} .
$$

In the above $\gamma=0.57721 \cdots=$ Euler's constant and

$$
h_{m}=1+\frac{1}{2}+\cdots+\frac{1}{m}, \quad h_{0}=1 .
$$

We assume the value of $\log (a x / 2)$ is known. Then, since expansions for $J_{k}(a x)$ and $I_{k}(a x)$ were found in Section 2 , and since $N_{k-1}(a x)$ is simply a polynomial in $1 /(a x)$, we need expand only the entire part of (4.3), i.e., $W_{k}(a x)$, in symmetric Jacobi polynomials.

Using the representation (4.6) as $f(x)$ in formula (2.5), a straight-forward derivation gives the series

$$
W_{k}(a x)=\sum_{n=0}^{\infty} A_{n} P_{n}{ }^{(\alpha, \alpha)}(x), \quad-1 \leqq x \leqq 1,
$$

where

$$
\begin{gathered}
A_{n}=\frac{\left[(-)^{k}+(-)^{n}\right](n+\alpha+1)_{\alpha}\left(n+\alpha+\frac{1}{2}\right)}{2^{n+2 \alpha+1}} \\
\cdot \sum_{m=0}^{\infty} \frac{(-)^{m}(-k-2 m)_{n}}{\left(m+\frac{k-n+1}{2}\right)_{n+\alpha+1}}\left(\frac{a}{2}\right)^{k+2 m} \frac{\left[h_{k+m}+h_{m}\right]}{m !(k+m) !} .
\end{gathered}
$$

We note that the expansion for $Y_{0}(a x)$ may also be obtained by partially differentiating (3.2) with respect to $v$ since

$$
Y_{0}(a x)=2 \pi^{-1}\left\{\frac{\partial J_{v}(a x)}{\partial v}\right\}_{v=0} .
$$

A similar procedure yields the expansion for $K_{0}(a x)$. The Jacobi series for $Y_{k}(a x)$ and $K_{k}(a x)$ for $k>0$, however, are not so easily obtained in this manner.

For $k=0$ and 1, the Chebyshev cases of (4.3) and (4.4) are

$$
\begin{array}{ll}
Y_{0}(a x)=\frac{2}{\pi}\left[\gamma+\ln \left(\frac{a x}{2}\right)\right] J_{0}(a x)+\sum_{n=0}^{\infty} E_{n} T_{2 n}(x), & 0<x \leqq 1 \\
Y_{1}(a x)=\frac{2}{\pi}\left[\gamma+\ln \left(\frac{a x}{2}\right)\right] J_{1}(a x)-\frac{2}{\pi a x}+\sum_{n=0}^{\infty} F_{n} T_{2 n+1}(x), & 0<x \leqq 1
\end{array}
$$




$$
\begin{array}{ll}
K_{0}(a x)=-\left[\gamma+\ln \left(\frac{a x}{2}\right)\right] I_{0}(a x)+\sum_{n=0}^{\infty} G_{n} T_{2 n}(x), & 0<x \leqq 1 \\
K_{1}(a x)=\left[\gamma+\ln \left(\frac{a x}{2}\right)\right] I_{1}(a x)+\frac{1}{a x}+\sum_{n=0}^{\infty} H_{n} T_{2 n+1}(x), & 0<x \leqq 1
\end{array}
$$

where

$$
\begin{aligned}
& E_{n}=\frac{2 \epsilon_{n}\left(\frac{a}{4}\right)^{2 n}(-)^{n+1} \sum_{k=0}^{\infty} \frac{(-)^{k}\left(\frac{a}{2}\right)^{2 k}\left(n+\frac{1}{2}\right)_{k} h_{n+k}}{\pi(n !)^{2}}}{(n+1)_{k}(2 n+1)_{k} k !} \\
& F_{n}=\frac{2(-)^{n+1}\left(\frac{a}{4}\right)^{2 n+1}}{\pi n !(n+1) !} \sum_{k=0}^{\infty} \frac{(-)^{k}\left(\frac{a}{2}\right)^{2 k}\left(n+\frac{3}{2}\right)_{k}\left[h_{n+k+1}+h_{n+k}\right]}{(n+2)_{k}(2 n+2)_{k} k !} \\
& G_{n}=\frac{\epsilon_{n}\left(\frac{a}{4}\right)^{2 n}}{(n !)^{2}} \sum_{k=0}^{\infty} \frac{\left(\frac{a}{2}\right)^{2 k}\left(n+\frac{1}{2}\right)_{k} h_{n+k}}{(n+1)_{k}(2 n+1)_{k} k !} \\
& H_{n}=-\frac{\left(\frac{a}{4}\right)^{2 n+1}}{n !(n+1) !} \sum_{k=0}^{\infty} \frac{\left(\frac{a}{2}\right)^{2 k}\left(n+\frac{3}{2}\right)_{k}\left[h_{n+k+1}+h_{n+k}\right]}{(n+2)_{k}(2 n+2)_{k} k !}
\end{aligned}
$$

5. Tables. Tables 1 through 3 are based on the Chebyshev polynomial cases of the expansions given in the previous sections of this paper. The entries in Tables 1 and 2 were computed on the UNIVAC 1103-A and those in Table 3 on the IBM 7090 at ASD. The calculations were designed so that the error incurred in using the expansions whose coefficients are tabulated here will not exceed five units in the 15th decimal place. Spot checks indicate the error is even less. Because all entries are to 16 significant figures, the expansions may be rearranged in powers of $x$ with no loss of accuracy.

The number in parentheses after each entry is the power of ten by which the entry is to be multiplied. We have chosen coefficients corresponding to $a=5$, but the coefficients for other values of $a$ from one through ten are available on request.

Note that the expansions in this paper are valid not only for $-1 \leqq x \leqq 1$ but for complex $x$ in a region which can be determined by a theorem of Szegö [1, p. 238]. More specifically, a Jacobi series representing an entire function converges everywhere in the finite complex plane. However, the further $x$ lies away from $-1 \leqq x \leqq 1$, the more the accuracy of the expansion deteriorates. This is so because $P_{n}^{(\alpha, \alpha)}(x)$ for complex $x$ can no longer be bounded by a simple power of $n$ but behaves in the following manner [10]

$$
\begin{aligned}
P_{n}{ }^{(\alpha, \alpha)}(z)=\frac{\Gamma(n+\alpha+1)}{n ! \pi^{1 / 2}} N^{2 \gamma}\left(\sin \frac{\theta}{2}\right)^{2 \gamma}\left(\cos \frac{\theta}{2}\right)^{-2 \gamma-2 \alpha-1} & \cdot \cos [N \theta+\pi \gamma]\left\{1+0\left(\frac{1}{N}\right)\right\}
\end{aligned}
$$

valid in the $z$ plane cut from -1 to $-\infty$ and from 1 to $\infty$. In (5.1), $\cos \theta=z$, 

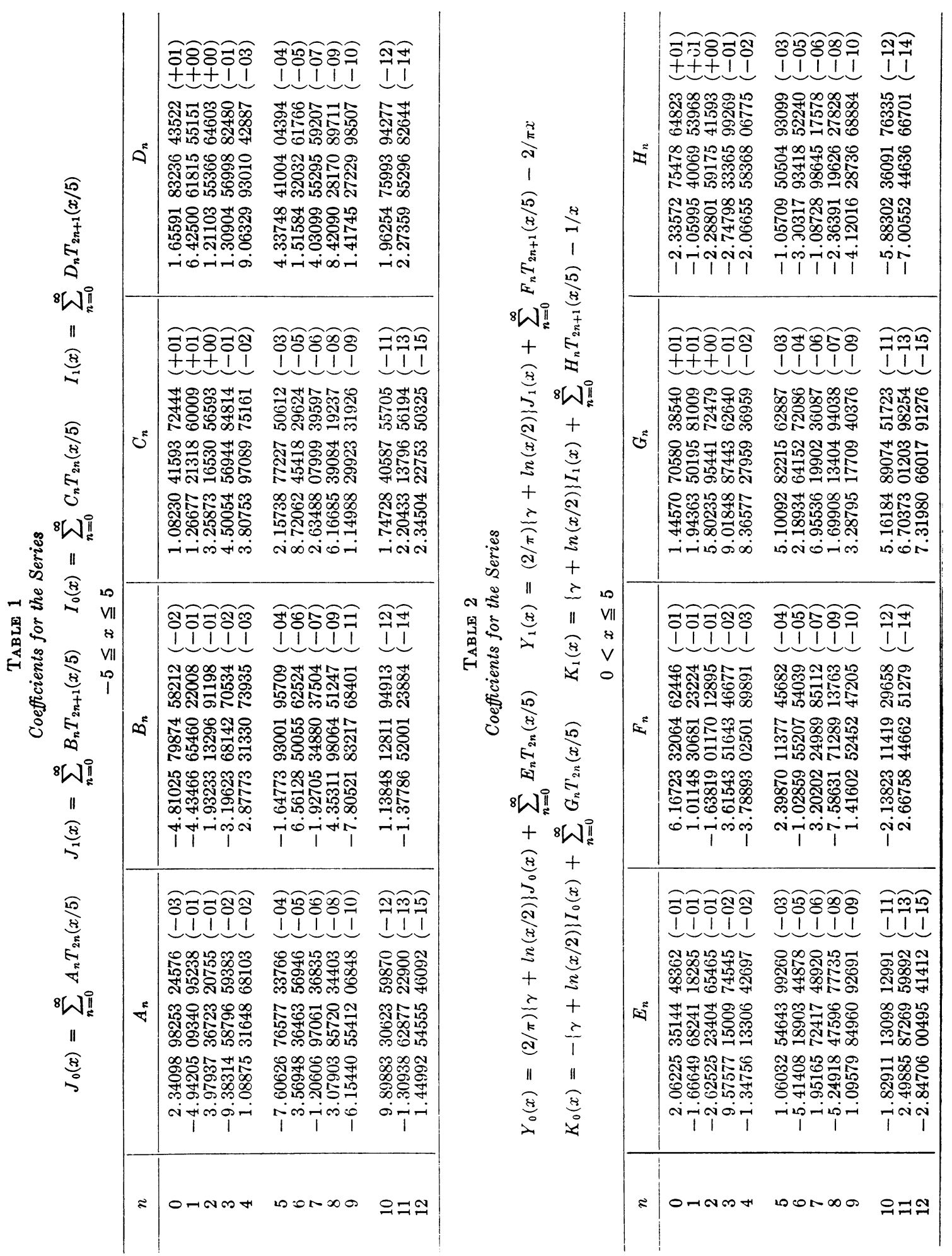


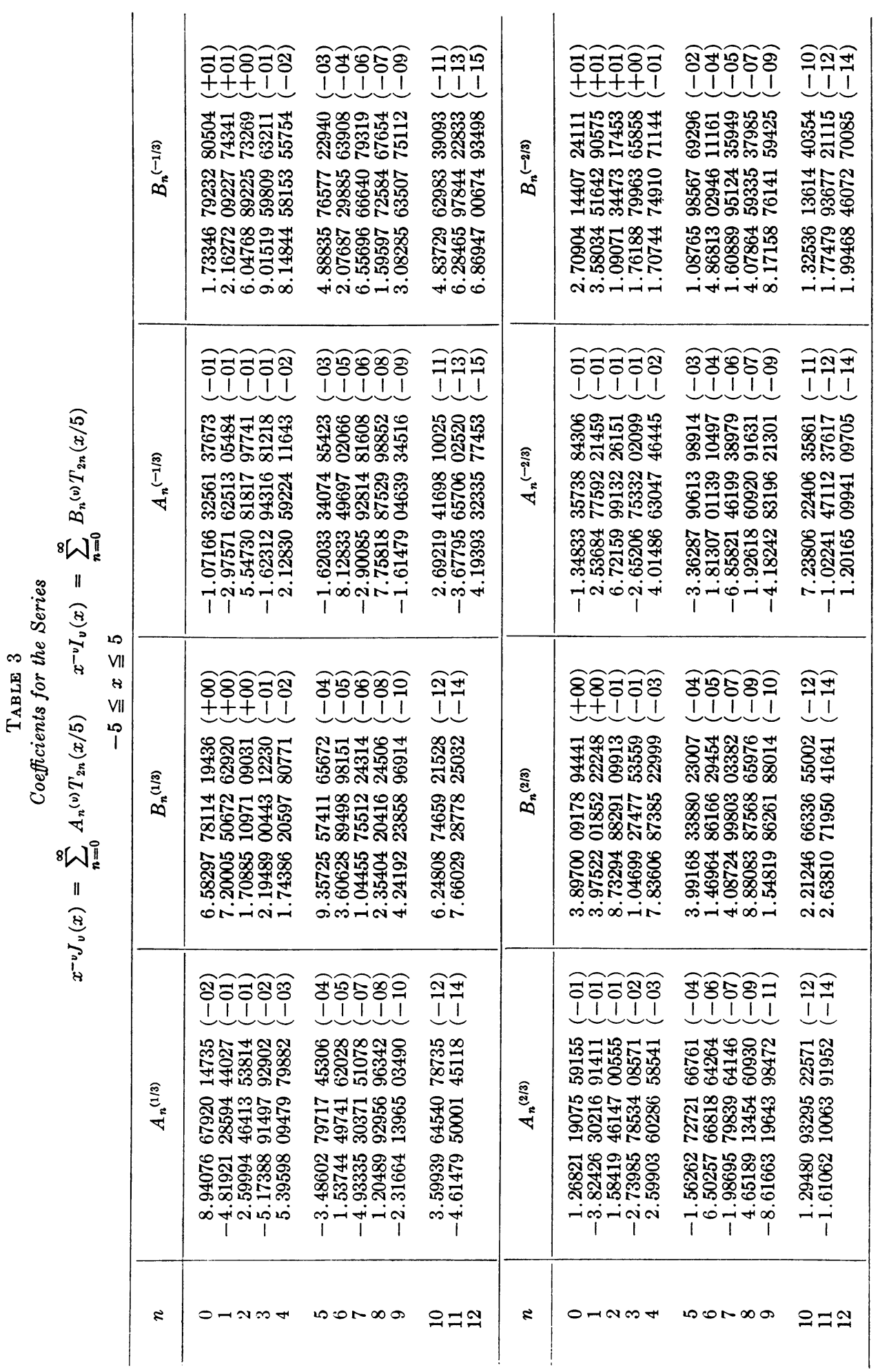




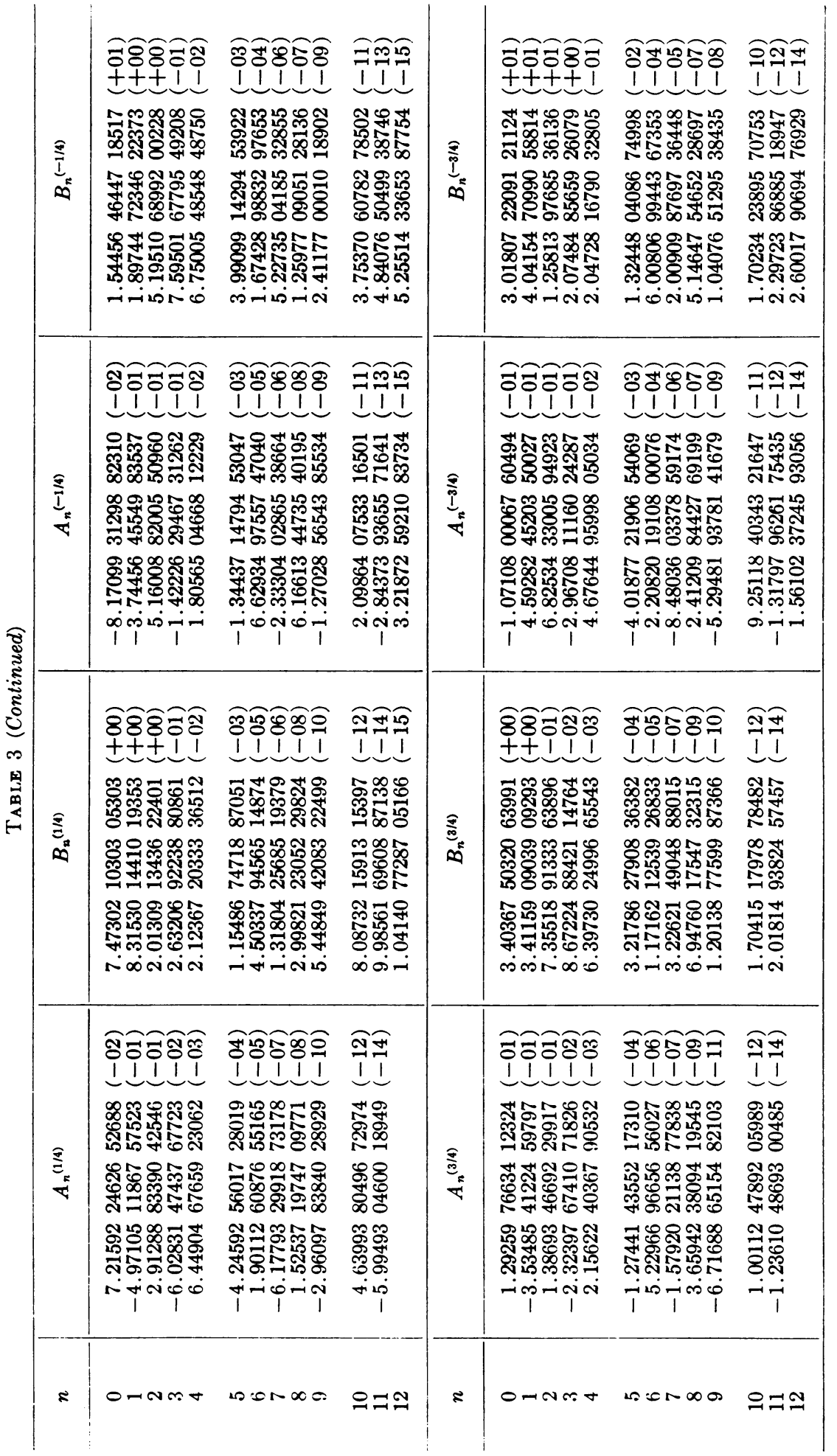


$N=[n(n+2 \alpha+1)]^{1 / 2}, \gamma=-(1+2 \alpha) / 4$. In general, if values of $f(x)$ for complex $x$ are desired, it is wisest to choose $a$ such that the expansions are interpolatory along a suitable ray in the complex $x$-plane and to stay as close as possible to this ray.

Suppose we have the truncated expansion

$$
f(x)=\sum_{n=0}^{N} A_{n} T_{n}(x)+\epsilon_{N+1}=\phi_{N}(x)+\epsilon_{N+1}, \quad-1 \leqq x \leqq 1,
$$

and

$$
\epsilon_{N+1}=\sum_{n=N+1}^{\infty} A_{n} T_{n}(x)
$$

Then $\phi_{N}(x)$ is not generally the Chebyshev approximation of degree $N$ to $f(x)$ in the sense of [11], i.e., the polynomial $\Phi_{N}(x)$ of degree $N$ uniquely characterized by the fact that in the interval $[-1,1]$ the number of consecutive points at which the difference $f(x)-\Phi_{N}(x)$ with alternate changes in sign assumes the value

$$
\max _{-1 \leqq x \leqq 1}\left|f(x)-\Phi_{N}(x)\right|,
$$

is not less than $N+2$; but $\phi_{N}(x)$ may closely approximate $\Phi_{N}(x)$. How closely, of course, depends on the coefficients $A_{n}$. If $A_{n}$ goes quite rapidly to zero as $n \rightarrow \infty$, then $A_{N+2}$ is small compared to $A_{N+1}$ and consequently

$$
\epsilon_{N+1} \sim A_{N+1} T_{N+1}(x)
$$

and the error curve is practically uniform, i.e., $\phi_{N}(x)$ is nearly $\Phi_{N}(x)$. Such is the case in our expansions, and, consequently, we must expect the approximation $\Phi_{N}(x)$ for moderate values of $a$ to offer a negligible improvement over the Chebyshev polynomial expansions derived in this paper and truncated after $N+1$ terms.

6. Acknowledgment. This work was supported by the United States Air Force through the Aeronautical Systems Division (ASD), Wright-Patterson Air Force Base, Ohio. The coefficients tabulated in this paper, as well as many others, were computed there, and the author gratefully acknowledges the programming skills of Messrs. Ralph Graham and Hank Bryson of ASD. Special thanks are due to Yudell Luke for his helpful suggestions and comments.

Midwest Research Institute

Kansas City, Missouri

1. G. Szegö, Orthogonal Polynomials, American Mathematical Society Colloquium Publications, v. XXIII, revised edition, New York, 1959.

2. A. Erdelyi, W. Magnus, F. Oberhettinger, \& F. G. Tricomi, Higher Transcendental Functions, v. 1 and 2 , McGraw-Hill, New York, 1953; v. 2, p. 168.

3. W. Gröb Ner, \& N. Hofreiter, Integraltafel, Zweiter Teil, Bestimmte Integrale, SpringerVerlag, Wein and Innsbruck, 1950, p. 189, No. (11a).

4. JerRy L. Fields, \& JeT Wimp, "Expansions of hypergeometric functions in hypergeometric functions," Math. Comp., XV, p. 390-395.

5. Nat. Bur. Standards ApPl. Math. Ser. No. 9, Tables of Chebyshev Polynomials, U.S. Government Printing Office, Washington, 1952; introduction by C. Lanczos, p. XI, No. (27).

6. I. A. Stegun, \& M. Abramowitz, "Generation of Bessel functions on high speed computers," $M T A C$, v. XI, p. 255-257. 
7. M. Goldstein, \& R. M. Thaler, "Recurrence techniques for the calculation of Bessel functions," $M T A C$, v. XIII, p. 102-108.

8. F. J. Corbat6, \& J. L. Unetsky, "Generation of spherical Bessel functions in digital computers," J. Assoc. Comp. Mach., VI, p. 366-375.

9. C. W. Clenshaw, "A note on the summation of Chebyshev series," MTAC, v. IX, p. 118-120.

10. Jerry L. Fields, \& Yudell L. Luke, "Asymptotic expansions of a class of hypergeometric polynomials with respect to the order I," to appear.

11. N. I. AcHIESER, Theory of Approximation, Ungar, New York, 1956, p. 57. 